\title{
Investigating rectal toxicity associated dosimetric features with deformable accumulated rectal surface dose maps for cervical cancer radiotherapy
}

Jiawei Chen ${ }^{1}$, Haibin Chen ${ }^{1}$, Zichun Zhong ${ }^{3}$, Zhuoyu Wang ${ }^{4}$, Brian Hrycushko ${ }^{2}$, Linghong Zhou ${ }^{1 *}$, Steve Jiang ${ }^{2}$, Kevin Albuquerque ${ }^{2}$, Xuejun $\mathrm{Gu}^{2^{*}}$ and Xin Zhen ${ }^{*^{*}}$ (D)

\begin{abstract}
Background: Better knowledge of the dose-toxicity relationship is essential for safe dose escalation to improve local control in cervical cancer radiotherapy. The conventional dose-toxicity model is based on the dose volume histogram, which is the parameter lacking spatial dose information. To overcome this limit, we explore a comprehensive rectal dose-toxicity model based on both dose volume histogram and dose map features for accurate radiation toxicity prediction.
\end{abstract}

Methods: Forty-two cervical cancer patients treated with combined external beam radiotherapy (EBRT) and brachytherapy (BT) were retrospectively studied, including 12 with Grade $\geq 2$ rectum toxicity and 30 patients with Grade 0-1 toxicity (non-toxicity patients). The cumulative equivalent 2-Gy rectal surface dose was deformably summed using the deformation vector fields obtained through a recent developed local topology preserved non-rigid point matching algorithm. The cumulative three-dimensional (3D) dose was flattened and mapped to a two-dimensional (2D) plane to obtain the rectum surface dose map (RSDM). The dose volume parameters (DVPs) were calculated from the 3D rectum surface, while the texture features and the dose geometric parameters (DGPs) were extracted from the 2D RSDM. Representative features further computed from DVPs, textures and DGPs by principle component analysis (PCA) and statistical analysis were respectively fed into a support vector machine equipped with a sequential feature selection procedure. The predictive powers of the representative features were compared with the GEC-ESTRO dosimetric parameters $D_{0.1 / 1 / 2 \mathrm{~cm}^{3}}$.

Results: Satisfactory predictive accuracy of sensitivity 74.75 and $84.75 \%$, specificity 72.67 and $79.87 \%$, and area under the receiver operating characteristic curve (AUC) 0.82 and 0.91 were respectively achieved by the PCA features and statistical significant features, which were superior to the $D_{0.1 / 1 / 2 \mathrm{~cm}^{3}}$ (AUC 0.71). The relative area in dose levels of 64Gy, 67Gy, 68Gy, 87Gy, 88Gy and 89Gy, perimeters in dose levels of 89Gy, as well as two texture features were ranked as the important factors that were closely correlated with rectal toxicity.

Conclusions: Our extensive experimental results have demonstrated the feasibility of the proposed scheme. A future large patient cohort study is still needed for model validation.

Keywords: Rectum toxicity prediction, Machine learning, Dose accumulation, Deformable registration, Cervical cancer

\footnotetext{
*Correspondence: smart@smu.edu.cn; Xuejun.Gu@utsouthwestern.edu; xinzhen@smu.edu.cn

${ }^{1}$ School of Biomedical Engineering, Southern Medical University, Guangzhou

510515, Guangdong, China

${ }^{2}$ Department of Radiation Oncology, The University of Texas, Southwestern

Medical Center, Dallas, TX 75390, USA

Full list of author information is available at the end of the article
}

(c) The Author(s). 2018 Open Access This article is distributed under the terms of the Creative Commons Attribution 4.0 International License (http://creativecommons.org/licenses/by/4.0/), which permits unrestricted use, distribution, and reproduction in any medium, provided you give appropriate credit to the original author(s) and the source, provide a link to the Creative Commons license, and indicate if changes were made. The Creative Commons Public Domain Dedication waiver (http://creativecommons.org/publicdomain/zero/1.0/) applies to the data made available in this article, unless otherwise stated. 


\section{Background}

The combination of the external beam radiotherapy (EBRT) and brachytherapy (BT) $(\mathrm{EBRT}+\mathrm{BT})$ is a common therapy regime for locally advanced cervical cancer [1]. Recent monocentric and multicentric EBRT+BT studies [2-4] have shown promising results with high tumor local control rate. However, radiation induced side effect (or toxicity) on organs at risk (OARs), such as rectum, bladder and vagina, is still a concern. Serious side effects such as bowel obstruction can occur months to years after treatment and impact negatively on the patients' quality-of-life. The correlation between OARs' morbidity and radiation dose parameters was analyzed in EMBRACE study [2]. Particularly, the $\mathrm{D}_{0.1 \mathrm{~cm}}{ }^{3}, \mathrm{D}_{1 \mathrm{cc}}$ and $\mathrm{D}_{2 \mathrm{~cm}}{ }^{3}$ of rectum were used to establish dose-toxicity relationship in the occurrence of rectal morbidity. $\mathrm{D}_{0.1 / 1 / 2 \mathrm{~cm}}{ }^{3}$ are conventional dose volume parameters (DVPs) extracted from dose volume histogram (DVH). Inherently, they are in deficiency of dosimetric spatial information. Studies have shown close relationship between the spatial dose characteristics and rectal toxicity [5-12]. For instance, Wortel et al. [12] observed significant differences in local rectal dose distribution between prostate cancer patients with and without toxicity by utilizing the unfolded two dimensional (2D) rectum surface dose map (RSDM). Similarly, Munbodh et al. [10] demonstrated that late rectal toxicity was related to dose on the upper rectum region by investigating dose pattern on the RSDM. Buettner et al. [6] analyzed the RSDM and found significant correlation between the subjective sphincter control and the dose delivered to the anal sphincter region. Another issue with current $\mathrm{D}_{0.1 / 1 / 2 \mathrm{~cm}}{ }^{3}$ evaluation procedure is that the cumulative dose is summed with an assumption that the hotspot regions are stationary throughout the entire fractional treatments [13, 14]. However, this static assumption is often violated by the large inter-fraction rectum deformation, especially in intra-cavity brachytherapy treatment cases [15-17]. Recently, promising advancements have been reported by Moulton et al. who investigated the associations between RSDM and gastrointestinal toxicities after deformably registering each phase of a combined EBRT-BT prostate cancer treatment [18]. These limited but inspiring studies shed light on the possibility of revealing more accurate dose-toxicity relationship by exploring the spatial dose distribution patterns on the deformable accumulated dose.

In this study, we proposed and evaluated a rectum dose-toxicity prediction scheme using both dose volume parameters and dose map spatial information. In addition, the accumulated rectal dose maps are obtained with the aid of an accurate deformable image registration. The accumulated 3D rectal surface dose was flattened to obtain a 2D RSDM. The DVPs were extracted from the DVHs of cumulative dose, while the texture features and the dose geometric parameters (DGPs) were extracted from the 2D
RSDM. Representative features further computed from DVPs, textures and DGPs by principle component analysis (PCA) and statistical analysis were respectively feed into a support vector machine (SVM) equipped with a sequential feature selection (SFS) procedure. The predictive powers of the representative features were compared with the GEC-ESTRO dosimetric parameters $\mathrm{D}_{0.1 / 1 / 2 \mathrm{cc}}$.

\section{Methods}

\section{Patient cohort}

Forty-two cervical cancer patients were retrospectively studied. These patients were treated with EBRT and BT. EBRT treatment plans were generated on the Pinnacle treatment planning system (Philips Medical Systems, Andover, MA, US) with 4-field box 3D plans or 9 field intensity modulation radiotherapy (IMRT) plans. EBRT plans were delivered with a total dose of 45Gy delivered in 25 fractions (1.8Gy per daily fraction). BT treatment boost were planned on Eclipse treatment planning system (Varian Medical Systems, Palo Alto, US). The BT boost plans were delivered immediately followed by the EBRT treatment with total dose of 28Gy in 4 fractions (7Gy per fraction and two fractions per week) or 30Gy in 5 fractions (6Gy per fraction and two fractions per week). The collected data include planning images and treatment plans. The patient was scheduled for follow-up examination every $2 \sim 3$ months after treatment. Patients complaining of hematochezia were further examined by colonoscopy. Twelve patients scored as Grade $\geq 2$ rectal toxicity per CTCAE v4 [19] were characterized as toxicity patients, and the other 30 Grade 0-1 patients were non-toxicity patients. To account for biologic effects of different fractionation schemes, both the rectum physical doses received in $\mathrm{BT}$ and EBRT were converted to EQD2 doses using a linear quadratic model [20] with an $\alpha / \beta$ ratio of 3 for dose summation [21, 22]. This retrospective study was approved by the institutional review board (IRB).

\section{Deformable dose accumulation and rectum unfolding}

For all patients, the volume of rectum was defined as the total rectal wall segmented between the level of the ischial tuberosity and the rectosigmoid junction, with a length ranging from $6 \sim 9 \mathrm{~cm}$ in the patient cohort. The rectum surface meshes were generated using rectum contours via a particle-based surface meshing approach [23].

A previously developed local topology preserved nonrigid registration point matching algorithm (TOP-DIR) was employed for rectum surface registration [24]. Details of the TOP-DIR algorithm can be found in Additional file 1: Appendix A. We regarded the first BT fraction as the reference and registered the other BT fractions rectum surface to the reference fraction rectum surface to obtain the 
deformation vector fields (DVFs), which were used to deform and sum fractional BT rectal doses to yield cumulative BT rectal dose. Considering a homogenous dose distribution often covers the entire pelvic region in our EBRT treatment plan regimen, we assumed a homogenous EBRT dose in the pelvic region and added the EBRT dose to the BT cumulated dose without deformation to obtain the total EBRT+BT dose.

The EBRT+BT rectal dose was then flattened via a 3D-2D mapping to generate a 2D RSDM. The 3D-2D rectal dose mapping is detailed in Additional file 1: Appendix B.

\section{Dosimetric features extraction}

Three types of dosimetric features, DVPs, texture feature and DGPs were extracted. The DVPs (21 in total) were $\mathrm{D} x$-cc (minimum dose in the most exposed $x$ - $\mathrm{cm}^{3}$ volume, $x \in[0.1,10]$ with $0.5 \mathrm{~cm}^{3}$ intervals) calculated from the $3 \mathrm{D}$ EBRT+BT rectal dose. The texture features (43 in total) were extracted from the RSDM, including 3 first-order gray level statistical global features, 9 Gy level co-occurrence matrix (GLCM) texture features, 13 Gy level run-length matrix (GLRLM) texture features, 13 Gy level size zone matrix (GLSZM) texture features, and 5 neighborhood gray-tone difference matrix (NGTDM) texture features [25]. The DGPs (224 in total) were computed from the RSDM at various dose levels, ranging from 45Gy to 100Gy with 1 Gy interval, including 1) the relative area (\%) of the dose region with respect to the area of rectum surface in the RSDM; 2) the perimeter (mm) of the dose region; 3 ) the relative width (\%), the ratio between the maximum width of the dose region with respect to the rectum circumference on the corresponding CT slice; and 4) the length $(\mathrm{mm})$ of the dose region. The DGPs are illustrated in Fig. 1.

\section{Toxicity prediction scheme}

We employed the support vector machine (SVM) [26] based method as our prediction scheme. A sequential forward feature selection (SFS) algorithm [27] was used to select a subset of features with best SVM prediction from the feature set (detailed in Additional file 1: Appendix C). We refer the above predictive scheme as SVM-SFS hereafter in this paper.

Considering the unbalanced training patient cohort where the toxicity group constitutes only a relative small portion of the dataset in this study, a synthetic minority over-sampling technique (SMOTE) [28] was used. The SMOTE balances the training dataset by over-sampling the minority class via introducing synthetic examples along the line segments joining $k$ minority class nearest neighbors. This data balancing technic has been shown to be helpful for avoiding over-fitting and better model generalization [29-33]. In the five-fold cross validation in this study, data balancing was merely applied to the training dataset in each fold of validation, while the validation dataset was kept unchanged for its "purity".

\section{Quantification and comparison}

The rectum surface registration accuracy is measured by four similarity metrics [24, 34], including the Dice's coefficient $(\mathrm{DC})$, the percent error $(\mathrm{PE})$, the mean vertex to vertex distance (VVD), and the Hausdorff distance (HD). Higher DC or lower PE, VVD, and HD indicate better results.

The predictive performance was quantified by the accuracy (ACC), sensitivity (SEN), specificity (SPE), and the area under the receiver operating characteristic (ROC) curve (AUC). An AUC of 0.5 is expected if a random prediction is performed. ACC, SEN and SPE are defined as: $A C C=(T P+T N) /(T P+F P+F N+T N)$, $\mathrm{SEN}=T P /(T P+F N)$ and $\mathrm{SPE}=T N /(T N+F P)$, where $T P$ is true positive, $T N$ is true negative, $F P$ is false positive and $F N$ is false negative. The mean ACC, SEN, SPE and AUC via a repeated (100 times) 5 -fold cross validation was reported.
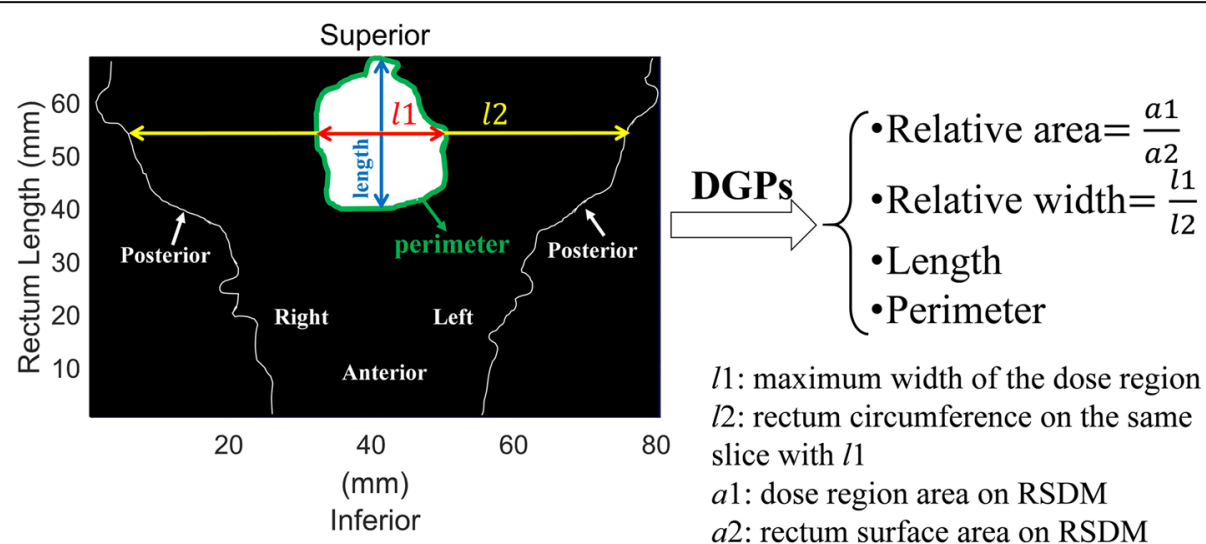

l1: maximum width of the dose region

12: rectum circumference on the same

slice with $l 1$

$a 1$ : dose region area on RSDM

$a 2$ : rectum surface area on RSDM

Fig. 1 Example of DGPs extracted from the RSDM at a certain dose level 
For a comparison study, the conventional dose volume parameters $D_{0.1 / 1 / 2 \mathrm{~cm}}{ }^{3}$ calculated via the "static-hotspot assumption" (SA) approach [35] were used as a baseline (referred as $\mathrm{SA}-\mathrm{D}_{0.1 / 1 / 2 \mathrm{~cm}}{ }^{3}$ ). The $\mathrm{SA}-\mathrm{D}_{0.1 / 1 / 2 \mathrm{~cm}^{3}}$ were compared to the features computed from the deformably summed EBRT+BT EQD2 dose on 3D rectum surfaces (DVPs) and flattened 2D RSDMs (texture features and DGPs). With many DVP and DGP parameters, it is possible to cause overfitting. To guard against overfitting, we extract representing features from DVPs and DGPs with 1) principle component analysis (PCA), and 2) statistical analyses (referred as $\mathbf{F}_{\mathbf{P C A}}$ and $\mathbf{F}_{\text {sta }}$, respectively). The $\mathbf{F}_{\mathbf{P C A}}$ were features in the PCA domain that calculated by performing the PCA on all the DVPs, texture features and DGPs with the first $n$ principal components account for $>99 \%$ of the variance. The $\mathbf{F}_{\text {sta }}$ were computed by performing statistical analyses (Mann-Whitney $\mathrm{U}$ test with raw $p$-values reported) on each feature category of DVPs, texture features and DGPs between the toxicity and non-toxicity groups, to screen out those statistical significant features.

The prediction capabilities of SA-D $\mathrm{D}_{0.1 / 1 / 2 \mathrm{~cm}}{ }^{3}, \mathbf{F}_{\mathbf{P C A}}$ and $\mathbf{F}_{\text {sta }}$ were compared by respectively feeding them into the SVM-SFS. The Z-test ( $p$-values were adjusted by the Bonferroni correction) was used for ROC curves comparisons, and all the statistical analyses conducted in this study were considered significant if $p<0.05$.

\section{Results}

\section{Rectum DIR}

The TOP-DIR was demonstrated to be robust for different rectum DIR scenarios, as seen in three example cases (Fig. 2a) with small, large and complex deformation. For all the evaluated cases, 156 DIRs were performed, and the
DC, PE, VVD and HD over the patient groups are depicted in Fig. 2b. Significant improvements were achieved after TOP-DIR point matching, with the median of DC increased from 0.71 to $0.86(p<0.001)$, the median of PE, VVD and HD decreased from 0.60, $1.53 \mathrm{~mm}$ and $6.52 \mathrm{~mm}$ to $0.26(p<0.001), 0.74 \mathrm{~mm}(p<0.001)$ and $4.06 \mathrm{~mm}(p<0.001)$, respectively.

\section{Representative features $\mathrm{F}_{\mathrm{PCA}}$ and $\mathrm{F}_{\text {sta }}$}

The computed $\boldsymbol{F}_{\boldsymbol{P C A} \boldsymbol{A}}$ were 10 representative PCA features that the first $n=10$ principal components were used. While the $\mathbf{F}_{\text {sta }}$ were statistical significant $(p<0.05)$ features of DVPs, texture features and DGPs, which were identified via the Mann-Whitney $U$ test. These significant features $\mathbf{F}_{\text {sta }}$ (73 in total) included: (1) 13 DVPs from $\mathrm{D}_{0.1 \mathrm{~cm}}{ }^{3}$ to $\mathrm{D}_{6 \mathrm{cc}}$; (2) 6 texture features: correlation in GLCM, long run high gray-level emphasis (LRHGE) in GLRLM, low gray-level zone emphasis (LGZE), high gray-level zone emphasis (HGZE) and small zone high gray-level emphasis(SZHGE) in GLSZM, complexity in NGTDM; (3) 54 DGPs, including relative areas in dose levels of 55Gy 64Gy, 67Gy 68Gy, 87Gy 89Gy; perimeters in dose levels of 54Gy 65Gy, 87Gy 89Gy; relative widths in dose level of $87 \mathrm{~Gy} \sim 89 \mathrm{~Gy}$; and length in dose levels of 59Gy 60Gy, 63Gy 64Gy, 66Gy 73Gy, 79Gy 83Gy, $85 \mathrm{~Gy}, 87 \mathrm{~Gy} 89 \mathrm{~Gy}$.

Prediction comparisons of $S A-D_{0.1 / 1 / 2 \mathrm{~cm}^{3}}, \mathrm{~F}_{\mathrm{PCA}}$ and $\mathrm{F}_{\text {sta }}$ The prediction performance of SA- $\mathrm{D}_{0.1 / 1 / 2 \mathrm{~cm}}{ }^{3}, \mathbf{F}_{\mathbf{P C A}}$ and $\mathbf{F}_{\text {sta }}$ were listed in Table 1. For traditional SA- $\mathrm{D}_{0.1 / 1 / 2 \mathrm{~cm}^{3}}$, the prediction resulted in SEN 66.25\%, SPE 66.73\%, and AUC 0.71 (95\% confidence interval [CI]: 0.68-0.72). For the $\mathbf{F}_{\mathbf{P C A}}$, better prediction performances were observed when compared with SA- $\mathrm{D}_{0.1 / 1 / 2 \mathrm{~cm}}{ }^{3}(p<0.001)$, with SEN

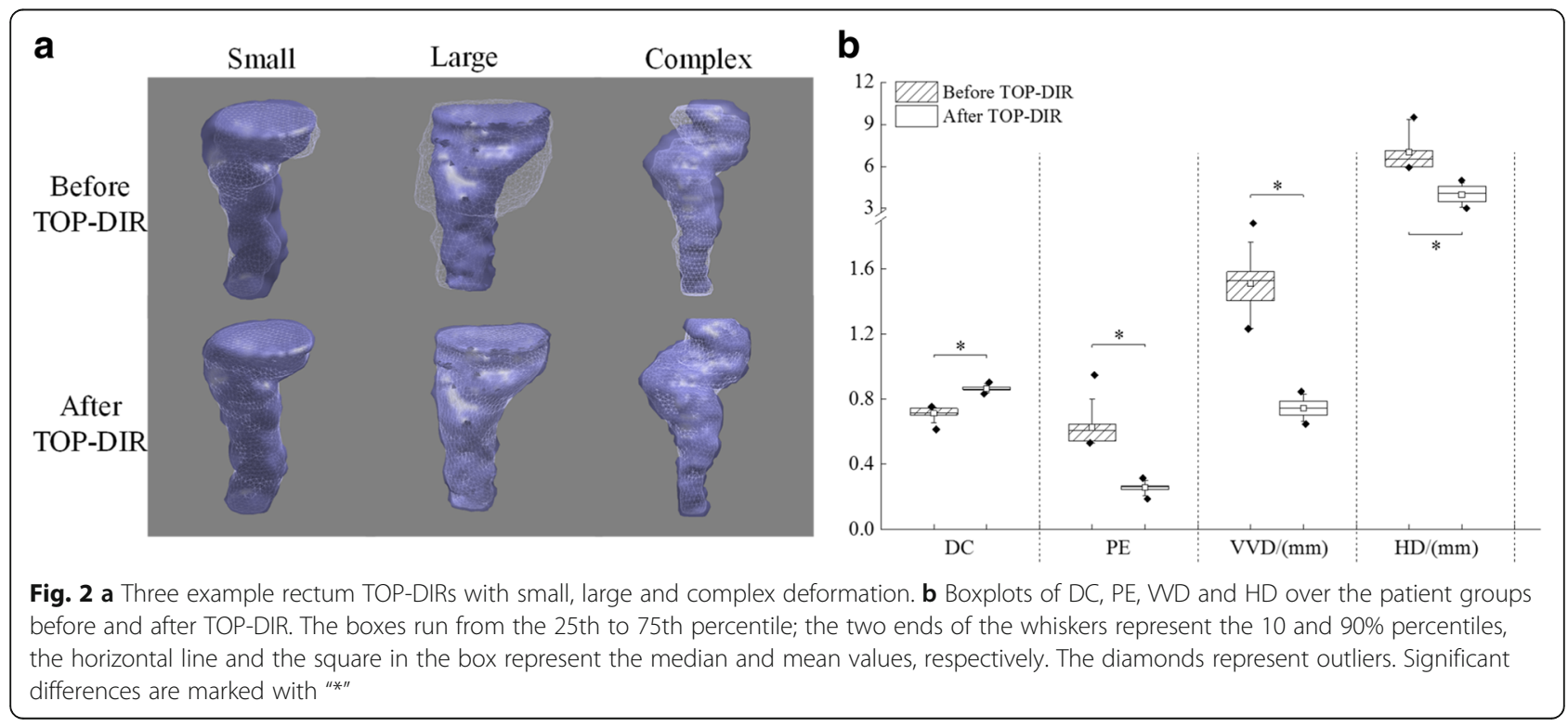


Table 1 SVM-SFS prediction on different features

\begin{tabular}{|c|c|c|c|c|c|}
\hline Features & & SEN & SPE & ACC & AUC $(95 \% \mathrm{Cl})$ \\
\hline$S A-D_{0.1 / 1 / 2 \mathrm{~cm}^{3}}$ & & $66.25 \%$ & $66.73 \%$ & $66.55 \%$ & $0.71(0.68-0.72)$ \\
\hline $\mathrm{F}_{\mathrm{PCA}}$ & & $74.75 \%$ & $72.67 \%$ & $73.22 \%$ & $0.82(0.75-0.85)$ \\
\hline \multirow[t]{7}{*}{$\mathrm{F}_{\text {sta }}$} & DVPs & $63.42 \%$ & $73.20 \%$ & $70.37 \%$ & $0.76(0.69-0.80)$ \\
\hline & Texture & $75.50 \%$ & $73.23 \%$ & $73.86 \%$ & $0.82(0.75-0.86)$ \\
\hline & DGPs & $60.42 \%$ & $74.53 \%$ & $70.46 \%$ & $0.79(0.72-0.81)$ \\
\hline & DVPs + Texture & $81.00 \%$ & $78.60 \%$ & $79.36 \%$ & $0.88(0.84-0.91)$ \\
\hline & DVPs + DGPs & $61.92 \%$ & $73.83 \%$ & $70.40 \%$ & $0.79(0.72-0.82)$ \\
\hline & DGPs + Texture & $85.17 \%$ & $79.13 \%$ & $80.84 \%$ & $0.91(0.85-0.92)$ \\
\hline & DVPs + Texture + DGPs & $84.75 \%$ & $79.87 \%$ & $81.32 \%$ & $0.91(0.87-0.93)$ \\
\hline
\end{tabular}

74.75\%, SPE 72.67\%, and AUC 0.82 (95\% CI: 0.75-0.85). While for $\mathbf{F}_{\text {sta }}$, we compared different combinations of the significant features of DVPs, texture features and DGPs in $\mathbf{F}_{\text {sta }}$. It is observed that using the DVPs alone in $\mathbf{F}_{\text {sta }}$ had only limited improvement when compared with SA-D $\mathrm{D}_{0.1 / 1 / 2 \mathrm{~cm}^{3}}(p=0.025)$. In contrast, using the texture features in $\mathbf{F}_{\text {sta }}$ or the DGPs in $\mathbf{F}_{\text {sta }}$ achieved better predictive performances than both the SA- $\mathrm{D}_{0.1 / 1 / 2 \mathrm{~cm}}{ }^{3}$ and DVPs in $\mathbf{F}_{\text {sta. }}$. The best predictive results were achieved by using the combinations of "DGPs + texture" or "DVPs + DGPs + texture" when compared with SA-D $\mathrm{D}_{0.1 / 1 / 2 \mathrm{~cm}^{3}}(p<$ 0.001 ), with SEN $85.17 \% / 84.75 \%$, SPE $79.13 \% / 79.87 \%$ and AUC 0.91 (95\% CI: 0.85-0.92)/0.91 (95\% CI: 0.87-0.93). The comparisons of SA- $\mathrm{D}_{0.1 / 1 / 2 \mathrm{~cm}^{3}}, \mathbf{F}_{\mathbf{P C A}}$ and $\mathbf{F}_{\text {sta }}$ via SVM-SFS were depicted by the ROC analysis in Fig. 3.

\section{Top ranked features statistics in $\mathrm{F}_{\text {sta }}$}

By utilizing all the 73 significant features of $\mathbf{F}_{\text {sta }}$, the SVM-SFS model was repeated 100 times and the features were ranked according to their frequencies of being selected.
The feature selection frequency distributions are shown in Fig. 4. The top-10 features included relative areas in dose levels of 64 Gy, 67Gy, 68Gy, 87Gy 89Gy, perimeters in dose levels of $89 \mathrm{~Gy}$, length in dose levels of $87 \mathrm{~Gy}$ and $88 \mathrm{~Gy}$, and two texture features: HGZE and complexity. No DVPs were ranked as the top-10 features.

Statitics of the top-10 features between the toxicity and non-toxicity groups are depicted in Table 2. For DGPs, the relative area in dose levels of 64Gy, 67Gy, 68Gy was significantly larger in the toxicity group $(p=$ 0.034, 0.049 and 0.045 , respectively). For the dose levels of 87Gy, 88Gy and 89Gy (with median $=0$ for both toxicity and non-toxicity groups), differences in distributions for the relative area were observed $(p=0.023,0.023$ and 0.023 , respectively). Similarly, the perimeter in dose levels of $89 \mathrm{~Gy}$ and the length in dose levels of 87Gy and 88Gy (all with median $=0$ for both toxicity and non-toxicity groups) have statistically significant differences in distributions between the two groups $(p=0.023, p=0.016$ and 0.023 , respectively). For texture features, the median (interquartile
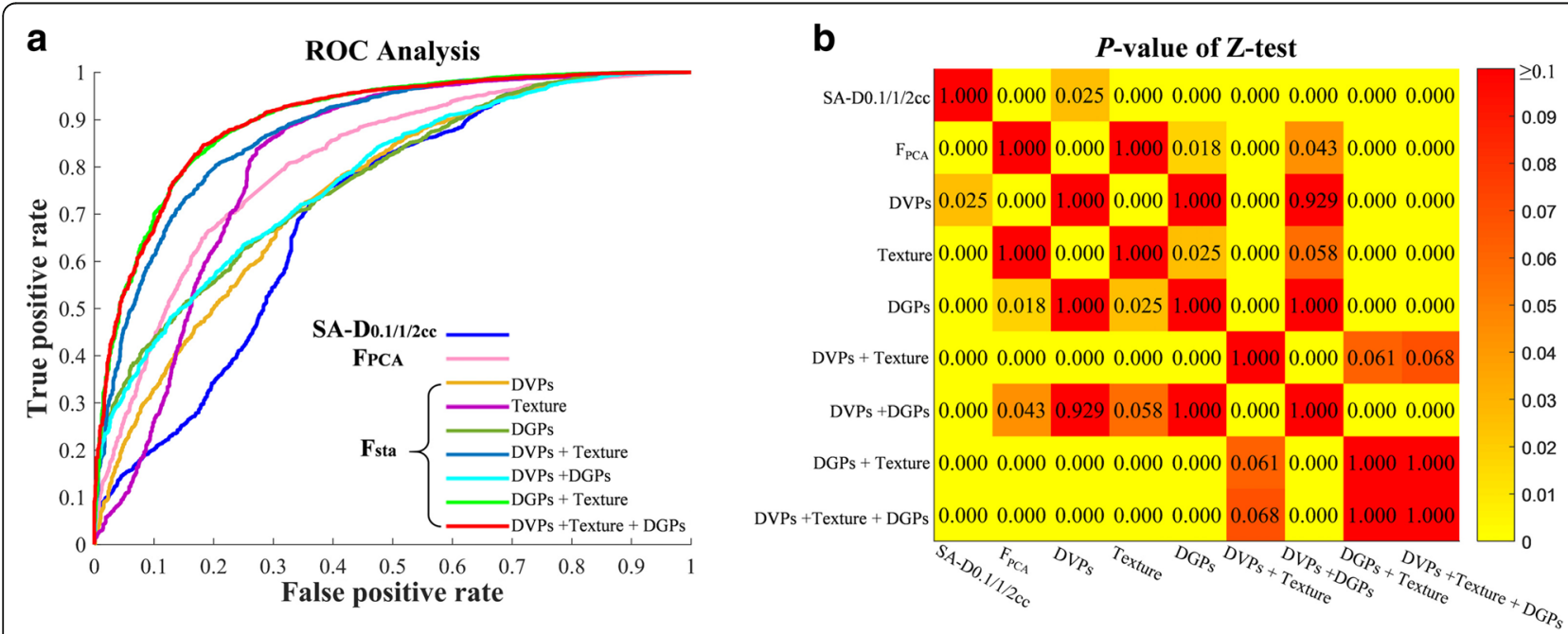

Fig. 3 a ROC analysis for different significant features and their combinations via SVM-SFS. b ROC curves comparisons via Z-test ( $p$-values were adjusted by the Bonferroni correction) 


\section{Feature Ranking}

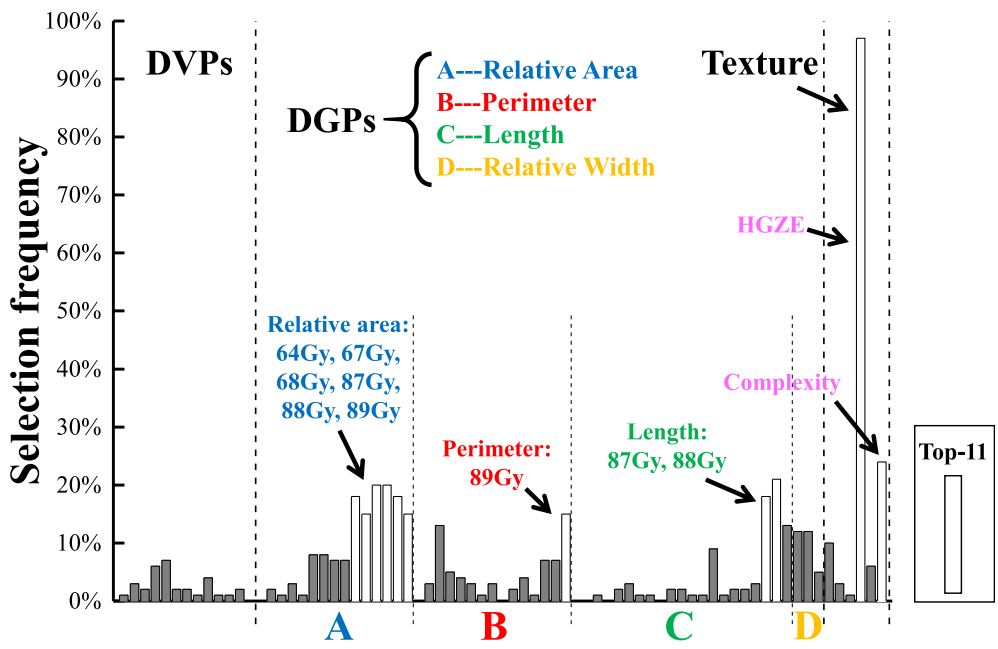

Fig. 4 Feature ranking via SVM-SFS (repeated 100 times) on feature combinations of "DVPs + Texture + DGPs" from $F_{\text {sta }}$

range, IQR) of HGZE were 317.81(51.19) vs. 376.63(37.70) $(p=0.0007)$, and the complexity were $130.71(9.04)$ vs. $119.77(10.18)(p=0.0087)$ between the two groups.

\section{Discussion}

An effective rectal toxicity prediction scheme is essential for guiding radiation treatment planning. $\mathrm{D}_{0.1 / 1 / 2 \mathrm{~cm}}{ }^{3}$ are recommended by the GEC-ESTRO guidelines [36] for rectum dose monitoring, however, their predictive capabilities for rectal toxicity are still under investigation. Other studies reported that the $D_{5 c c}$ may be a more reliable estimate than other dose volume parameters to predict risks of rectosigmoid mucosal changes and late rectal complications $[37,38]$. All these studies essentially used DVPs to predict rectal toxicity. The findings in current work align with the previous studies, e.g., statistically significant differences were observed in DVPs ranging between $D_{0.1 \mathrm{~cm}}{ }^{3} \sim D_{6 c c}$. However, better prediction was accomplished by utilizing all the significant DVPs in $\mathbf{F}_{\text {sta }}$ when compared with merely using the SA- $\mathrm{D}_{0.1 / 1 / 2 \mathrm{~cm}^{3}}$ (Table 1$)$.

In this study, we have compared two approaches, i.e., the PCA analysis and the statistical analysis, in extracting representative features for feeding the SVM-SFS prediction scheme. The merit of performing PCA is to reduce the number and correlation of the potential features by converting the features into a set of values of linearly uncorrelated variables ( $\mathbf{F}_{\mathbf{P C A}}$ in this study). However, these converted values in the PCA domain carry no physical meanings, it is therefore difficult to interpret the prediction why the $\mathbf{F}_{\mathbf{P C A}}$ features are responsible for yielding corresponding prediction result. On the other hand, the statistical analysis approach reserves the physical meanings of the features by statistically pre-screening the significant features $\left(\mathbf{F}_{\text {sta }}\right.$ in this study), and the comparison evaluations also showed superior performance if all the features in $\mathbf{F}_{\text {sta }}$ were used for prediction, when compared with $\mathbf{F}_{\mathbf{P C A}}$.

Recently, researchers started to investigate the prediction model with spatial dose information. For instance, Buettner et al. presented a late rectal toxicity method based on the parameterized representation of the 3D rectal dose [39]. Lee et al. proposed a metric based on both surface dose and distance to predict incidence of the rectal bleeding in prostate cancer patients treated with radical radiotherapy [40]. Drean et al. identified rectal subregions at risk of rectal bleeding by performing voxel-wise analysis on the rectal dose distribution [7]. In this study, we took advantage of the hollow structure of the rectum and flattened the 3D

Table 2 Statistical analysis of the top 10 features in $\mathbf{F}_{\text {sta }}$ between the toxicity and non-toxicity groups

\begin{tabular}{|c|c|c|c|c|c|c|c|c|c|c|c|c|}
\hline & & \multicolumn{9}{|l|}{ DGPs } & \multirow{2}{*}{\multicolumn{2}{|c|}{ Texture }} \\
\hline & & \multicolumn{6}{|c|}{ Relative area (\%) } & \multirow{2}{*}{$\begin{array}{l}\text { Perimeter } \\
(\mathrm{mm}) \\
89 \mathrm{~Gy}\end{array}$} & \multicolumn{2}{|c|}{ Length (mm) } & & \\
\hline & & 64Gy & 67Gy & $68 \mathrm{~Gy}$ & $87 \mathrm{~Gy}$ & $88 \mathrm{~Gy}$ & 89Gy & & $87 \mathrm{~Gy}$ & $88 G y$ & HGZE & Complexity \\
\hline \multirow[t]{2}{*}{ Median(IQR) } & Toxi & $25.26(15.57)$ & $19.07(20.57)$ & $16.87(20.22)$ & $0(0.3)$ & $0(0.12)$ & $0(0.05)$ & $0(9.77)$ & $0(3.5)$ & $0(1.75)$ & 317.81 (51.19) & $130.71(9.04)$ \\
\hline & Non-toxi & 16.87 (16.59) & $12.26(18.29)$ & $9.48(16.28)$ & $0(0)$ & $0(0)$ & $0(0)$ & $0(0)$ & $0(0)$ & $0(0)$ & 376.63 (37.70) & $119.77(10.18)$ \\
\hline \multicolumn{2}{|l|}{ P-Value } & 0.034 & 0.049 & 0.045 & 0.023 & 0.023 & 0.023 & 0.023 & 0.016 & 0.023 & 0.001 & 0.009 \\
\hline
\end{tabular}


rectal dose to 2D RSDM to establish dose map toxicity prediction scheme. Though the RSDM neglects the doses in the rectum thickness direction, it preserves spatial dose information. The texture features and the DGPs, which are crafted to capture spatial dose distribution characteristics from the 2D RSDM, are able to provide more geometric and positional dosimetric information. Pioneer studies have shown potential correlations of spatial dose characteristics with rectal toxicity. For example, Drean et al. reported that the rectal subregions at risk of rectal bleeding are primarily located in the subprostatic anterior hemi-rectum and upper part of the anal canal [7]. Kim et al. found substantial correlation between rectal toxicity and percent rectal circumference at certain dose levels. Similarly, in this study, we have seen texture features and the geometric dosimetric features had better predictive power than the DVPs. These results hint us that rectum's response to dose might be dose-spatial dependent. As shown in Table 2 and Fig. 4, geometric feature such as the relative area, the perimeter and the length were found to be associated with rectal toxicity. The toxicity group tended to have larger dose coverage on the high dose region (64, 67 and 68Gy). This finding was in agreement with previous studies that rectal bleeding was significantly correlated with high-dose metrics [25, 41-43]. In addition, although only four DGPs were investigated in this work, other DGPs which were explored in previous studies also indicated associations with rectal toxicity. For instance, Buettner et al. investigated the eccentricity of the fitted ellipse of the dose region and found associations of the eccentricity with loose stools $[5,39,44]$. Moulton et al. also reported that compactness, circularity and confinement to the ellipse fits were correlated with rectal bleeding [18]. Incorporating these spatial features, which are crafted to depict the dose coverage and the shape of dose distribution, into the current model may potentially improve the predictive performance. Besides, two texture features, i.e., the HGZE and Complexity, were statistically different between the two groups, where the toxicity group has lower HGZE value (the Median(IQR) 317.81(51.19) vs. 376.63(37.70), $p=0.0007$ ) but higher Complexity value (the Median(IQR) 130.71(9.04) vs. 119.77(10.18), $p=$ 0.0087). However, how these texture features impact on rectal toxicity is still unclear. We applied the texture features on the RSDM with the intention to describe localized dosimetric patterns on the RSDM which are usually difficult to be noticed by human eyes. Yet, the drawbacks of the texture features are their deficiency of physical implications on interpreting correlations. Moreover, feature stability, e.g., whether the selected texture feature will change if different patient cohort size are used, is still an exploratory issue [45-47]. Since current work is a pioneer feasibility study of applying texture feature analysis on the deformably accumulative rectum surface dose map, more in-depth investigations on a larger patient cohort is still required in the future.

In this study, the generated 2D RSDM reserves the physical length of the rectum in both the superior-inferior direction and the circumferential direction on each slice (see Additional file 1: Appendix B). This was to ensure that the geometric features (e.g., area, perimeter, length, etc.) extracted from the RSDM would carry physical implications to signify the scale of dose delivered on rectum surface. Note that the drawback for reserving the physical dimension of the rectum is that the inter-patient variations of the rectum size could possibly influence/mask the significances of differences of the extracted geometric features between the two groups, especially given a small patient cohort. But the rectum size tends to be a random number across patients and therefore its impact would decrease and be minor in a larger patient cohort.

In addition, reporting accurate accumulated dose over the entire treatment course is a nontrivial task because of the substantial inter-fractional rectum deformation exists in the BT treatments. In this work, a previously developed TOP-DIR algorithm was used, although accurate geometric registration accuracy had been achieved and validated on a porcine bladder phantom $(\sim 2 \mathrm{~mm})$, further phantom studies are still needed to justify its effectiveness in rectum registration, and the dosimetric errors in the subsequent dose summation step also need to be monitored.

In this study, we added the EBRT dose to the accumulated BT dose without deformation. The reasons are twofold: firstly, a homogenous dose distribution (hot spot < $107 \%$ ) often covers the entire pelvic region in our EBRT treatment plan regimen. Often large portion of rectum are within treatment fields, especially for 3D plans. Only a very small inferior portion of the rectum is outside of the large pelvis treatment fields and dose variation across rectum is often within $15 \%$. With this relative homogenous dose in a large region across the pelvis, we could assume rectum receiving a consistent and homogenous dose in EBRT as planned. In this study, the EBRT plans were generated with 4-field box 3D plans or 9 field IMRT plans. Theoretically, these two techniques on a same patient would result in different EBRT dose distributions due to dose conformity and hence different accumulated dose on RSDM. It is therefore more appropriate to investigate the extracted features for each technique. However, it is impractical to implement in current study since only a small patient sample was available. The influence of these two EBRT techniques on the stability of the extracted features still needs further investigations on a larger patient cohort.

On the other hand, DIR between EBRT and BT CTs is challenging because of the clinical use of the intracavitary applicator in BT. Registering the BT CT image with applicator to the EBRT CT image without applicator (or vice versa) is difficult, if not impossible, since the point-to-point 
correspondence assumption is usually violated in most DIR algorithms. Consequently, the dosimetric uncertainties via EBRT-BT DIR might be possibly even larger than that summed without deformation. There are several reported attempts to address this issue [48, 49], for example, Berendsen et al. [48] proposed a DIR with penalty term that minimizes the volume of the missing structure for cervical MR images with and without applicator. Vasquez Osorio et al. [49] validated a structurewise registration with vector field integration to map the largely deformed anatomies between EBRT and BT. However, the EBRT-BT DIR needs to be treated prudently, and these novel methods need comprehensive validations before they can be confidently applied in a clinical setting. Adding EBRT to BT without deformation is therefore a reasonable approximation without knowing the uncertainties brought by the EBRT-BT DIR.

The choice of prediction models and feature selection strategies may also affect the predictive performance. We used the SVM-SFS scheme because it is the most common method to construct a predictive model with simultaneously feature selection. Though satisfactory performances have been achieved, other predictive models (e.g., random forest classifier) or feature selection methods (e.g., clonal selection algorithm) can provide even better predictive accuracy [50, 51].

For screening of the representative feature $F_{\text {sta }}$, the unadjusted $p$-values were used for statistical analysis, however, the current findings will probably change if the $p$-values were corrected for multiple testing. In fact, $p$-value adjustment is restrictive to application with many tests and applying it in the context of RSDM analysis is still controversial $[18,52]$. Since the physical length of the rectum was reserved on the RSDM in this study, the resolutions of the RSDMs were essentially patient specific. Multiple testing might not be applicable for this scenario where the resolution of the RSDM is fixed for each patient. Even though $p$-values corrections have been reported in other similar investigations using RSDM for rectal toxicity studies, however, the adjusted $p$-values did not demonstrate clear trends across regions on the RSDM where only limited and isolated regions of significance were found after applying multiple testing correction in RSDM analysis [18, 52, 53]. Furthermore, reporting the raw $p$-values is an exploratory study of finding predictive factors correlated to rectal toxicity without the risk of missing important factors which might be discarded if found to be insignificant after $p$-value adjustment [18].

One limitation of current study is that the patient cohort was small. Thus, the number of extracted features was larger than the patient sample size. To reduce the chance of getting over-fitting, a statistical analysis was performed to screen out the significant features before feeding into the predictive model. This guarantees a more robust feature ranking in the subsequent feature selection step in SVM-SFS. But note that a more effective way to observe overfitting is to separate the patient cohort into three datasets, i.e., one for training, one for validation and hyper-parameter tuning and one for testing. However, it was impossible to effectively separate our samples into three datasets, and cross validation was therefore our secondary option for model performance observation in a small patient cohort. But overfitting might also occur in the cross-validation space attributed to other factors such as the quantity of features considered, the selection of model hyper-parameters, etc., therefore, larger patient data is a key for evaluating model stability and generalization capability.

Another limitation of the study is that our study is purely on dosimetric parameter without consider clinical factors. Multivariable modeling of radiotherapy outcomes has been conducted by El Napa et al. [54]. We will further include clinical factors in our near future studies.

\section{Conclusions}

In summary, we have proposed and validated a rectum toxicity prediction method based on an accurate point registration and machine learning for cervical cancer radiotherapy. The extensive experimental results have demonstrated the feasibility of the proposed scheme for rectal toxicity prediction, rendering it a potential tool for clinical OARs dose control and complication prediction.

\section{Additional file}

Additional file 1: Appendix A. TOP-DIR algorithm. Appendix B. 3D-2D rectum surface dose mapping. Appendix $C$. Sequential forward feature selection (SFS) algorithm. (DOCX $155 \mathrm{~kb}$ )

\footnotetext{
Abbreviations

ACC: accuracy; AUC: area under the receiver operating characteristic curve; BT: brachytherapy; DC: the Dice's coefficient; DGPS: dose geometric parameters; DVFs: deformation vector fields; DVH: dose volume histogram; DVPs: dose volume parameters; EBRT: external beam radiotherapy; FN: false negative; FP: false positive; $F_{P C A}$ : representative PCA features extracted from DVPs, textures and DGPs; $F_{\text {sta: }}$ : representative (statistically significant) features screened out from DVPs, textures and DGPs; GLCM: gray level co-occurrence matrix; GLRLM: gray level run-length matrix; GLSZM: gray level size zone matrix; HD: the Hausdorff distance; HGZE: high gray-level zone emphasis; IRB: institutional review board; LGZE: low gray-level zone emphasis; LRHGE: long run high gray-level emphasis; NGTDM: neighborhood gray-tone difference matrix; OARs: organs at risk; PCA: principle component analysis; PE: the percent error; RBF: radial basis function; ROC curve: receiver operating characteristic curve; RSDM: rectum surface dose map; SA: static-hotspot assumption; $S A-D_{0.1 / 1 / 2 \mathrm{~cm}^{3}}$ : dose volume parameters $D_{0.1 / 1 / 2 \mathrm{~cm}}{ }^{3}$ calculated using the "static-hotspot assumption" addition method; SEN: sensitivity; SFS: sequential forward feature selection; SMOTE: synthetic minority over-sampling technique; SPE: specificity; SVM: support vector machine; SZHGE: small zone high gray-level emphasis; TN: true negative; TOP-DIR: topology preserved point matching-deformable image registration; TP: true positive; WD: the mean vertex to vertex distance
} 


\section{Funding}

This work is supported in part by the Varian Medical Systems, Inc. (\#OTD-109235), the National Science Foundation (NSF) ACl-1657364, the National Natural Science Foundation of China (81728016 and 81571771), the Guangdong Natural Science Foundation (2015A030313302), and the Science and Technology Planning Project of Guangdong Province, China (2015A020214013).

\section{Availability of data and materials}

The datasets analyzed during the current study are available from the corresponding author on reasonable request.

\section{Authors' contributions}

The manuscript was written through contributions of all authors. $X Z, X G$ and LZ designed the study. JC, HC and XZ collected all the data and performed the experiments and analysis. ZZ generated all the rectum surface meshes. JC implemented the SVM-SFS model and wrote the manuscript. ZW, BH, LZ, SJ, KA discussed the results and revised the manuscript. All authors have given approval to the final version of the manuscript.

\section{Ethics approval and consent to participate}

This retrospective patient study was approved by Human Research Protection Program Office (HRPPO)/Institutional Review Board (IRB) of The University of Texas Southwestern Medical Center. All methods in this study were conducted in accordance with the relevant guidelines and regulations. Considering that this is not a therapeutical treatment study, our institutional review board waived the need for obtaining written informed consent from the participants.

\section{Consent for publication}

Not applicable.

\section{Competing interests}

The authors declare that they have no competing interests.

\section{Publisher's Note}

Springer Nature remains neutral with regard to jurisdictional claims in published maps and institutional affiliations.

\section{Author details}

${ }^{1}$ School of Biomedical Engineering, Southern Medical University, Guangzhou 510515, Guangdong, China. ${ }^{2}$ Department of Radiation Oncology, The University of Texas, Southwestern Medical Center, Dallas, TX 75390, USA. ${ }^{3}$ Department of Computer Science, Wayne State University, Detroit, MI 48202, USA. ${ }^{4}$ Department of Epidemiology, Biostatistics and Occupational Health, McGill University, 805 Sherbrooke Street West, Montreal, Quebec H3A 0G4, Canada.

\section{Received: 17 October 2017 Accepted: 18 June 2018}

\section{Published online: 06 July 2018}

\section{References}

1. Monk BJ, Tewari KS, Koh W-J. Multimodality therapy for locally advanced cervical carcinoma: state of the art and future directions. J Clin Oncol. 2007; 25:2952-65

2. Tanderup K, Fokdal LU, Sturdza A, Haie-Meder C, Mazeron R, van Limbergen E, Jurgenliemk-Schulz I, Petric P, Hoskin P, Dorr W, et al. Effect of tumor dose, volume and overall treatment time on local control after radiochemotherapy including MRI guided brachytherapy of locally advanced cervical cancer. Radiother Oncol. 2016;120(3):441-6.

3. Mazeron R, Fokdal LU, Kirchheiner K, Georg P, Jastaniyah N, Šegedin B, Mahantshetty U, Hoskin P, Jürgenliemk-Schulz I, Kirisits C, et al. Dose-volume effect relationships for late rectal morbidity in patients treated with chemoradiation and MRI-guided adaptive brachytherapy for locally advanced cervical cancer: results from the prospective multicenter EMBRACE study. Radiother Oncol. 2016;120:412-9.

4. Kirchheiner K, Nout RA, Lindegaard JC, Haie-Meder C, Mahantshetty U, Segedin B, Jurgenliemk-Schulz IM, Hoskin PJ, Rai B, Dorr W, et al. Dose-effect relationship and risk factors for vaginal stenosis after definitive radio(chemo)therapy with image-guided brachytherapy for locally advanced cervical cancer in the EMBRACE study. Radiother Oncol. 2016;118:160-6.
5. Buettner F, Gulliford SL, Webb S, Sydes MR, Dearnaley DP, Partridge M. Assessing correlations between the spatial distribution of the dose to the rectal wall and late rectal toxicity after prostate radiotherapy: an analysis of data from the MRC RT01 trial (ISRCTN 47772397). Phys Med Biol. 2009;54:6535-48.

6. Buettner F, Gulliford SL, Webb S, Sydes MR, Dearnaley DP, Partridge M. The dose-response of the anal sphincter region-an analysis of data from the MRC RT01 trial. Radiother Oncol. 2012;103:347-52.

7. Dréan G, Acosta O, Ospina JD, Fargeas A, Lafond C, Corrégé G, Lagrange JL, Créhange $G$, Simon A, Haigron P. Identification of a rectal subregion highly predictive of rectal bleeding in prostate cancer IMRT. Radiother Oncol. 2016; 119:388-97.

8. Heemsbergen WD, Hoogeman MS, Hart GA, Lebesque JV, Koper PC Gastrointestinal toxicity and its relation to dose distributions in the anorectal region of prostate cancer patients treated with radiotherapy. Int J Radiat Oncol Biol Phys. 2005;61:1011-8.

9. Meijer GJ, van den Brink M, Hoogeman MS, Meinders J, Lebesque JV. Dosewall histograms and normalized dose-surface histograms for the rectum: a new method to analyze the dose distribution over the rectum in conformal radiotherapy. Int J Radiat Oncol Biol Phys. 1999;45:1073-80.

10. Munbodh R, Jackson A, Bauer J, Schmidtlein CR, Zelefsky MJ. Dosimetric and anatomic indicators of late rectal toxicity after high-dose intensity modulated radiation therapy for prostate cancer. Med Phys. 2008;35:2137-50.

11. Tucker SL, Zhang M, Dong L, Mohan R, Kuban D, Thames HD. Cluster model analysis of late rectal bleeding after IMRT of prostate cancer: a case-control study. Int J Radiat Oncol Biol Phys. 2006;64:1255-64.

12. Wortel RC, Witte MG, van der Heide UA, Pos FJ, Lebesque JV, van Herk M, Incrocci L, Heemsbergen WD. Dose-surface maps identifying local dose-effects for acute gastrointestinal toxicity after radiotherapy for prostate cancer. Radiother Oncol. 2015;117:515-20.

13. Kirisits C, Pötter R, Lang S, Dimopoulos J, Wachter-Gerstner N, Georg D. Dose and volume parameters for MRI-based treatment planning in intracavitary brachytherapy for cervical cancer. Int J Radiat Oncol Biol Phys. 2005:62:901-11.

14. Jamema SV, Mahantshetty U, Tanderup K, Malvankar D, Sharma S, Engineer R, Chopra S, Shrivastava SK, Deshpande DD. Inter-application variation of dose and spatial location of volumes of OARs during MR image based cervix brachytherapy. Radiother Oncol. 2013;107:58-62.

15. Fokdal L, Tanderup K, Nielsen SK, Christensen HK, Røhl L, Pedersen EM, Schønemann NK, Lindegaard JC. Image and laparoscopic guided interstitial brachytherapy for locally advanced primary or recurrent gynaecological cancer using the adaptive GEC ESTRO target concept. Radiother Oncol. 2011;100:473-9.

16. Pötter R, Kirisits C, Fidarova EF, Dimopoulos JCA, Berger D, Tanderup K, Lindegaard JC. Present status and future of high-precision image guided adaptive brachytherapy for cervix carcinoma. Acta Oncol. 2008;47:1325-36.

17. Tanderup K, Georg D, Pötter R, Kirisits C, Grau C, Lindegaard JC. Adaptive Management of Cervical Cancer Radiotherapy. Semin Radiat Oncol. 2010;20: $121-9$.

18. Moulton CR, House MJ, Lye V, Tang Cl, Krawiec M, Joseph DJ, Denham JW, Ebert MA. Spatial features of dose-surface maps from deformably-registered plans correlate with late gastrointestinal complications. Phys Med Biol. 2017; 62:4118-39.

19. National Cancer Institute, National Institutes of Health, US Department of Health and Human Services. Common Terminology Criteria for Adverse Events (CTCAE) Version 4.0. Published May 28, 2009; Revised version 4.03 June 14, 2010 (Available from: http://evs.nci.nih.gov/ftp1/CTCAE/About.html).

20. Bentzen SM, Dorr W, Gahbauer R, Howell RW, Joiner MC, Jones B, Jones DT, van der Kogel AJ, Wambersie A, Whitmore G. Bioeffect modeling and equieffective dose concepts in radiation oncology-terminology, quantities and units. Radiother Oncol. 2012;105:266-8.

21. Michalski JM, Gay H, Jackson A, Tucker SL, Deasy JO. Radiation dose-volume effects in radiation-induced rectal injury. Int J Radiat Oncol Biol Phys. 2010; 76:S123-9.

22. Moulton CR, House MJ, Lye V, Tang Cl, Krawiec M, Joseph DJ, Denham JW, Ebert MA. Prostate external beam radiotherapy combined with high-dose-rate brachytherapy: dose-volume parameters from deformably-registered plans correlate with late gastrointestinal complications. Radiat Oncol. 2016;11:144.

23. Zhong Z, Guo X, Wang W, Vy B, Sun F, Liu Y, Mao W. Particle-based anisotropic surface meshing. ACM Trans Graph. 2013;32:99.

24. Haibin C, Zichun Z, Yuliang L, Arnold P, Brian H, Kevin A, Xin Z, Linghong Z, Xuejun G. A non-rigid point matching method with local topology 
preservation for accurate bladder dose summation in high dose rate cervical brachytherapy. Phys Med Biol. 2016;61:1217.

25. Schaake W, van der Schaaf A, van Dijk LV, Bongaerts AH, van den Bergh AC, Langendijk JA. Normal tissue complication probability (NTCP) models for late rectal bleeding, stool frequency and fecal incontinence after radiotherapy in prostate cancer patients. Radiother Oncol. 2016;119:381-7.

26. Cortes C, Vapnik V. Support-vector networks. Mach Learn. 1995;20:273-97.

27. Jain A, Zongker D. Feature selection: evaluation, application, and small sample performance. IEEE Trans Pattern Anal Mach Intell. 1997;19:153-8.

28. Chawla NV, Bowyer KW, Hall LO, Kegelmeyer WP. SMOTE: synthetic minority over-sampling technique. Comput Sci. 2011;

29. Kayalibay B, Jensen $G$, van der Smagt P. CNN-based segmentation of medical imaging data. arXiv preprint arXiv:170103056 2017 (Available from: https://arxiv.org/abs/1701.03056).

30. Milletari F, Navab N, Ahmadi S-A. V-net: Fully convolutional neural networks for volumetric medical image segmentation. In 3D Vision (3DV), 2016 Fourth International Conference on. IEEE; 2016: 565-571 (Available from: https:// ieeexplore.ieee.org/abstract/document/7785132/).

31. Ronneberger O, Fischer P, Brox T. U-net: Convolutional networks for biomedical image segmentation. In International Conference on Medical image computing and computer-assisted intervention. Springer; 2015: 234241 (Available from: https://link.springer.com/chapter/10.1007/978-3-31924574-4_28).

32. Chawla NV, Bowyer KW, Hall LO, Kegelmeyer WP. SMOTE: synthetic minority over-sampling technique. J Artif Int Res. 2002;16:321-57.

33. He H, Bai Y, Garcia EA, Li S. ADASYN: Adaptive synthetic sampling approach for imbalanced learning. In: 2008 IEEE International Joint Conference on Neural Networks (IEEE World Congress on Computational Intelligence); 2008. p. 1322-8.

34. Chen H, Zhen X, Gu X, Yan H, Cervino L, Xiao Y, Zhou L. SPARSE: seed point auto-generation for random walks segmentation enhancement in medical inhomogeneous targets delineation of morphological MR and CT images. J Appl Clin Med Phys. 2015;16:5324.

35. ICRU. ICRU REPORT 89: Prescribing, recording, and reporting brachytherapy for Cancer of the cervix. J ICRU. 2013;13

36. Pötter R, Haie-Meder C, Limbergen EV, Barillot I, Brabandere MD, Dimopoulos J, Dumas I, Erickson B, Lang S, Nulens A, et al. Recommendations from gynaecological (GYN) GEC ESTRO working group (II): concepts and terms in 3D image-based treatment planning in cervix cancer brachytherapy-3D dose volume parameters and aspects of 3D image-based anatomy, radiation physics, radiobiology. Radiother Oncol. 2006;78:67-77.

37. Kim TH, Kim J-Y, Sohn DK, Kim Y-J, Lee Y-S, Moon SH, Kim SS, Kim DY. A prospective observational study with dose volume parameters predicting rectosigmoidoscopic findings and late rectosigmoid bleeding in patients with uterine cervical cancer treated by definitive radiotherapy. Radiat Oncol. 2013;8:28.

38. Kim Y, Kim Y-J, Kim J-Y, Lim YK, Jeong C, Jeong J, Kim M, Lim MC, Seo S-S, Park S-Y. Toxicities and dose-volume histogram parameters of MRI-based brachytherapy for cervical cancer. Brachytherapy. 2017;16:116-25.

39. Buettner F, Gulliford SL, Webb S, Partridge M. Modeling late rectal toxicities based on a parameterized representation of the 3D dose distribution. Phys Med Biol. 2011;56:2103-18.

40. Lee R, Chan EK, Kosztyla R, Liu M, Moiseenko V. Dose-distance metric that predicts late rectal bleeding in patients receiving radical prostate externalbeam radiotherapy. Phys Med Biol. 2012;57:8297-307.

41. Ebert MA, Foo K, Haworth A, Gulliford SL, Kennedy A, Joseph DJ, Denham JW. Gastrointestinal dose-histogram effects in the context of dose-volumeconstrained prostate radiation therapy: analysis of data from the RADAR prostate radiation therapy trial. Int J Radiat Oncol Biol Phys. 2015;91:595-603.

42. Ospina JD, Zhu J, Chira C, Bossi A, Delobel JB, Beckendorf V, Dubray B, Lagrange JL, Correa JC, Simon A, et al. Random forests to predict rectal toxicity following prostate cancer radiation therapy. Int J Radiat Oncol Biol Phys. 2014;89:1024-31.

43. Munbodh R, Jackson A. Quantifying cell migration distance as a contributing factor to the development of rectal toxicity after prostate radiotherapy. Med Phys. 2014;41:021724.

44. Buettner F, Gulliford SL, Webb S, Partridge M. Using Bayesian logistic regression to evaluate a new type of dosimetric constraint for prostate radiotherapy treatment planning. Med Phys. 2010;37:1768-77.
45. Bologna M, Montin E, Corino VDA, Mainardi LT. Stability assessment of first order statistics features computed on ADC maps in soft-tissue sarcoma. In: 2017 39th Annual International Conference of the IEEE Engineering in Medicine and Biology Society (EMBC); 11-15 July 2017; 2017. p. 612-5.

46. Bogowicz M, Riesterer O, Bundschuh RA, Veit-Haibach $P$, Hullner M, Studer G, Stieb S, Glatz S, Pruschy M, Guckenberger M, Tanadini-Lang S. Stability of radiomic features in CT perfusion maps. Phys Med Biol. 2016;61:8736-49.

47. Larue RTHM, van Timmeren JE, de Jong EEC, Feliciani G, Leijenaar RTH, Schreurs WMJ, Sosef MN, Raat FHPJ, van der Zande FHR, Das M, et al. Influence of gray level discretization on radiomic feature stability for different $C T$ scanners, tube currents and slice thicknesses: a comprehensive phantom study. Acta Oncol. 2017;56:1544-53.

48. Berendsen FF, Kotte ANTJ, de Leeuw AAC, Viergever MA, Pluim JPW. Free-Form Registration Involving Disappearing Structures: Application to Brachytherapy MRI. In: Yoshida H, Warfield S, Vannier MW, editors. Abdominal Imaging Computation and Clinical Applications: 5th International Workshop, Held in Conjunction with MICCAI 2013, Nagoya, Japan, September 22, 2013 Proceedings. Berlin, Heidelberg: Springer Berlin Heidelberg; 2013. p. 136-44.

49. Vásquez Osorio EM, Kolkman-Deurloo I-KK, Schuring-Pereira M, Zolnay A Heijmen BJM, Hoogeman MS. Improving anatomical mapping of complexly deformed anatomy for external beam radiotherapy and brachytherapy dose accumulation in cervical cancer. Med Phys. 2015;42:206-20.

50. Khalilia M, Chakraborty S, Popescu M. Predicting disease risks from highly imbalanced data using random forest. BMC Med Inform Decis Mak. 2011;11:51.

51. Zhou Z, Folkert M, Cannon N, lyengar P, Westover $K$, Zhang Y, Choy H, Timmerman R, Yan J, Xie X-J, et al. Predicting distant failure in early stage NSCLC treated with SBRT using clinical parameters. Radiother Oncol. 2016;119:501-4.

52. Palorini F, Cozzarini C, Gianolini S, Botti A, Carillo V, lotti C, Rancati T, Valdagni R, Fiorino C. First application of a pixel-wise analysis on bladder dose-surface maps in prostate cancer radiotherapy. Radiother Oncol. 2016; 119:123-8.

53. Yahya N, Ebert MA, House MJ, Kennedy A, Matthews J, Joseph DJ, Denham JW. Modeling urinary dysfunction after external beam radiation therapy of the prostate using bladder dose-surface maps: evidence of spatially variable response of the bladder surface. Int J Radiat Oncol Biol Phys. 2017;97:420-6.

54. El Naqa I, Bradley J, Blanco Al, Lindsay PE, Vicic M, Hope A, Deasy JO. Multivariable modeling of radiotherapy outcomes, including dose-volume and clinical factors. Int J Radiat Oncol Biol Phys. 2006;64:1275-86.

\section{Ready to submit your research? Choose BMC and benefit from:}

- fast, convenient online submission

- thorough peer review by experienced researchers in your field

- rapid publication on acceptance

- support for research data, including large and complex data types

- gold Open Access which fosters wider collaboration and increased citations

- maximum visibility for your research: over $100 \mathrm{M}$ website views per year

At BMC, research is always in progress.

Learn more biomedcentral.com/submissions 